\title{
Non-linear weak lensing forecasts
}

\author{
Luciano Casarini, ${ }^{a, b}$ Giuseppe La Vacca, ${ }^{a, b}$ Luca Amendola, ${ }^{c}$ Silvio \\ A. Bonometto ${ }^{a, b}$ \& Andrea V. Macciò ${ }^{d}$ \\ ${ }^{a}$ Department of Physics G. Occhialini, Milano-Bicocca University \\ Piazza della Scienza 3, 20126 Milano, Italy \\ ${ }^{b}$ I.N.F.N., Sezione di Milano-Bicocca \\ Piazza della Scienza 3, 20126 Milano, Italy \\ ${ }^{c}$ Institute of Theoretical Physics \\ Philosophenweg 16, 69120 Heidelberg, Germany \\ ${ }^{d}$ Max-Planck-Institut für Astronomie \\ Königstuhl 17, 69117 Heidelberg, Germany \\ E-mail: luciano.casarini@mib.infn.it, giuseppe.lavacca@mib.infn.it, \\ l.amendola@thphys.uni-heidelberg.de, bonometto@oats.inaf.it,maccio@mpia.de
}

\begin{abstract}
We investigate the impact of non-linear corrections on dark energy parameter estimation from weak lensing probes. We find that using HALOFIT expressions, suited to $\Lambda \mathrm{CDM}$ models, implies substantial discrepancies with respect to results directly obtained from $N$-body simulations, when $w(z) \neq-1$. Discrepancies appear strong when using models with $w^{\prime}(z=0)>0$, as fiducial models; they are however significant even in the neighborhood of $\Lambda \mathrm{CDM}$, where neglecting the degrees of freedom associated with the DE state equation can lead to a misestimate of the matter density parameter $\Omega_{m}$.
\end{abstract}

Keywords: cosmology: theory, dark matter, gravitation; methods: numerical, N-body simulations. 


\section{Contents}

1 Introduction 1

2 Weak lensing parameter estimation $\quad 2$

3 Cosmological models $\quad 6$

$\begin{array}{lll}4 & \text { Simulations and their analysis } & 7\end{array}$

5 Predictions on parameter errors $\quad 9$

5.1 Derivatives 9

5.2 Likelihood ellipses: $\Lambda$ CDM neighborhood 11

5.3 Confidence ellipses: M1 \& M3 models 12

6 Conclusions

\section{Introduction}

The evidence in favor of dark energy (DE), a smooth component with largely negative state parameter $w \sim-1$, is more than a decade old. The original claim based on the acceleration in the expansion rate, deduced from the Hubble diagram of supernovae Ia [1], has been confirmed and strengthened by other probes, in particular cosmic microwave background (CMB) spectra (see, e.g., [2]) and large scale structure (LSS) data [3]. The nature of DE is however one of the major puzzles of today's physics and astrophysics. In order to investigate it, more data constraining the DE state equation are sought.

Data based on the CMB spectra $C_{\ell}$, however, only scarcely constrain the equation of state parameter of the Dark Energy component $w(z)$. In fact, although the integrated Sachs $\&$ Wolfe (ISW) effect depends on $w(z)$, it mostly affects low $-\ell C_{\ell}$ which are also affected by the $z$-dependence of the optical depth $\tau$. Furthermore, ISW is an integral effect, and low $-\ell$ spectral components are subject to a large cosmic variance.

Putting together CMB and LSS data is already more effective, as this tests the fluctuation distribution and amplitude at two different $z$ values. Clearly, estimating the fluctuation spectrum $P(k, z)=\left\langle\left|\delta_{m}(k, z)\right|^{2}\right\rangle$ at several $z$ values, so to work out the growth rate

$$
G(z, k)=\delta_{m}(k, z) / \delta_{m}(k, 0),
$$

would open a real window on $w(z)$ and, therefore, on DE nature. So far $G$ is only very weakly constrained (see, e.g., [4]), but a formidable array of surveys are being performed or planned to measure it through weak lensing (WL), high- $z$ power spectra and cluster counts [5].

This paper will deal with WL probes (see, e.g., [6] for a thorough review). The power of this tool is illustrated by the fact that, to fully exploit next generation WL surveys, we need predictions on non-linear power spectra accurate up to $\sim 1 \%[7]$.

Such precision goes beyond the claimed $\pm 3 \%$ accuracy of the popular HALOFIT expressions [8] based on the halo model of structure formation and calibrated using numerical simulations of $\Lambda \mathrm{CDM}$ models; they yield a map of linear $\kappa$ 's onto non-linear $k$ 's, so that $P_{n l}(k)=P_{\text {lin }}(\kappa)$. Leaving apart the need of a generic improvement of HALOFIT accuracy [9], 
previous work [10] already showed that, using HALOFIT expressions for non- $\Lambda C D M$ models, requires suitable corrections. In spite of that, the HALOFIT map has been often used to obtain the spectra of models with non-constant DE state parameter $w(z)$, that we shall denominate dynamical DE (dDE) models, herebelow. This procedure was dictated by the lack of appropriate extensions of HALOFIT to non $-\Lambda$ CDM cosmologies.

The aim of this paper is to quantify the effects of using non-linear dDE spectra obtained with the HALOFIT map, when the nature of DE is investigated through WL surveys as Euclid [11] or COSMOS [12].

To this aim we focus on dDE models with

$$
w(z)=w_{0}+w_{a}(1-a)=w_{0}+w_{a} z /(1+z)
$$

( $a$ being the scale factor), although bearing in mind that they are already a restricted class of $\mathrm{dDE}$ models, their state equations being fully characterized by amplitude and derivative at $z=0$. Through a Fisher Matrix (FM) approach, we evaluate the errors in WL estimates for $w_{0}, w_{a}$ and $\Omega_{m}$ (the matter density parameter). We do so using matter power spectra obtained either from HALOFIT or $N$-body simulations and then we compare the likelihood ellipses on the parameters obtained with the two approaches. To our knowledge, this is the first paper in which a Fisher matrix approach is performed directly on $N$-body outputs rather than on fits or other approximations.

We will assume that the shear field is observed in a survey which approximate the current design of Euclid [11]. This will be a survey of approximately half sky (20,000 square degrees) with galaxies at an average depth of $z_{m} \approx 0.9$ and 40 galaxies per square arcminute. The redshifts will be evaluated photometrically assuming a normal distribution with variance $\sigma_{z}=0.05$ for errors. The tests are performed assuming as fiducial cosmology $\Lambda$ CDM model $(w \equiv-1)$, as well as for two dDE models, still consistent with the CMB data from WMAP7 [2] and related data, roughly the most distant models from $\Lambda$ CDM that are still allowed by the data (at $95 \%$ confidence level (CL) ) in the $w_{o}-w_{a}$ plane (see figure 1 ). In this way we explore the dependence of our results on the assumed fiducial model. We can anticipate the conclusion that a straightforward use of HALOFIT is significantly misleading, in quite a few cases.

\section{Weak lensing parameter estimation}

The first step to estimate the errors in the parameter measured through weak lensing techniques, amounts to define the convergence weak lensing power spectrum, which in the linear regime is identical to the ellipticity power spectrum [6]. This spectrum is a linear function of the matter power spectrum convolved with the lensing properties of space. According to [13], if we bin the galaxies in $n_{b}$ redshift bins labeled by $i, j=1, \ldots n_{b}$, it reads

$$
P_{i j}(\ell)=H_{0}^{3} \int_{0}^{\infty} \frac{d z}{E(z)} W_{i}(z) W_{j}(z) P_{n l}\left(\frac{H_{0} \ell}{r(z)}, z\right) .
$$

Here $P_{n l}(k, z)$ is the non-linear matter power spectrum at redshift $z$ and the $W_{i}$ are window functions that will be explicitely given below. Through this paper $n_{b}=1,3$ and 5 will be considered, in order to assess the dependence of the results on the binning procedure (there is no much gain in going beyond 5 bins [14]). In the last two cases, the bin limits $z_{i}$ are selected so to have the same number of galaxies per bin. Let us then define the other quantities in this 


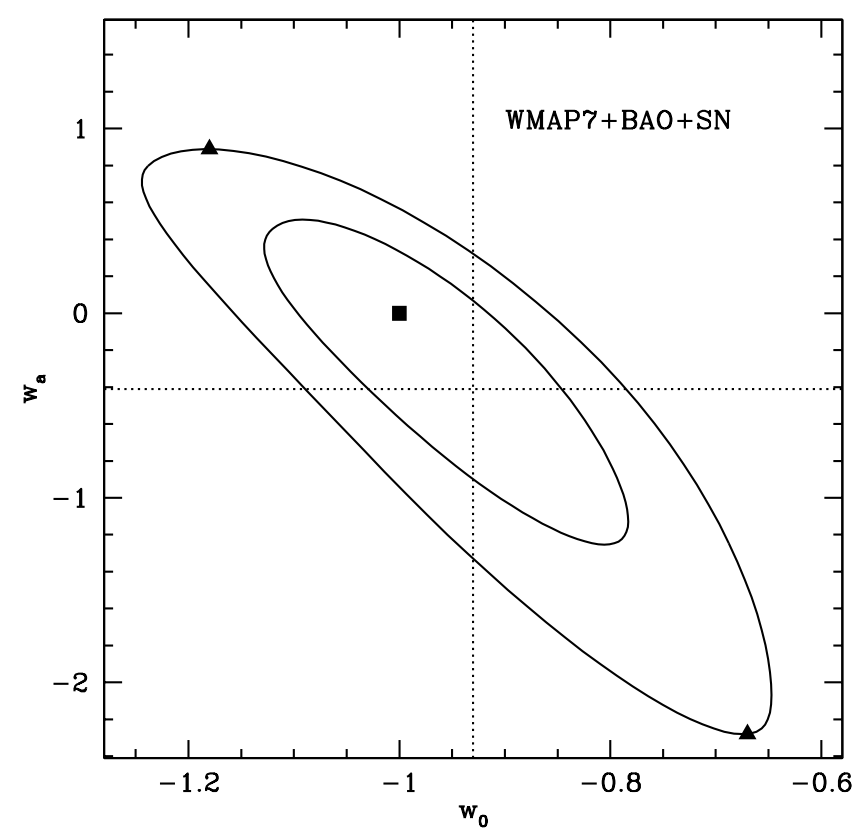

Figure 1. Constraints on the time-dependent DE equation of state (1.2), derived from WMAP7 spectra combined with SNIa and BAO updated data [2]. The contours show the $68 \%$ and $95 \%$ $\mathrm{CL}$. The square point indicate the $\Lambda \mathrm{CDM}$ model; the triangle points are the other two reference cosmologies considered in this work: the model with negative (positive) $w_{a}$ is also dubbed M1 (M3). The intersection of the dotted lines is the best fit point in [2] $w_{o}=-0.93, w_{a}=-0.41$.

expression, starting from the redshift dependence of the Hubble parameter $H(z) \equiv E(z) H_{0}$ and

$$
r\left(z_{1}, z_{2}\right)=\int_{z_{1}}^{z_{2}} \frac{d z}{E(z)} ;
$$

accordingly, by referring to the scale factor $a$ and the conformal time $\tau$, we have that

$$
d z / E(z)=H_{0}\left(-d a / a^{2}\right) /\left(\dot{a} / a^{2}\right)=-H_{0} d \tau,
$$

so that eq. (2.2) also reads

$$
r\left(z_{1}, z_{2}\right)=H_{0}\left(\tau_{1}-\tau_{2}\right) .
$$

From here we deduce that $r(z) \equiv r(0, z)=H_{0}\left[\tau_{0}-\tau(z)\right]$.

Let us then consider also the distribution of the galaxy number per unit redshift and solid angle. We assume the following simple parametrized form

$$
n(z)=d^{2} N / d \Omega d z=\mathcal{C}\left(z / z_{0}\right)^{A} \exp \left[-\left(z / z_{0}\right)^{B}\right] \quad \text { with } \quad \mathcal{C}=\left(B / z_{0}\right) \Gamma^{-1}[(A+1) / B]
$$

where $\Gamma(x)$ is the Gamma function. Here we choose $A=2, B=1.5$, so that $\mathcal{C}=1.5 / z_{0}$. If $z_{m}=0.9$ is the average redshift, one then has $z_{0}=z_{m} / 1.412$. This choice of values is a good approximation to the observed selection function of a survey like Euclid [11]. By integrating the distribution $n(z)$ across the depth of the $j$-th bin $\Delta z_{j}$ we have the angular density in steradians

$$
n_{j}=3600(180 / \pi)^{2} \int_{\Delta z_{j}} d z n(z)
$$




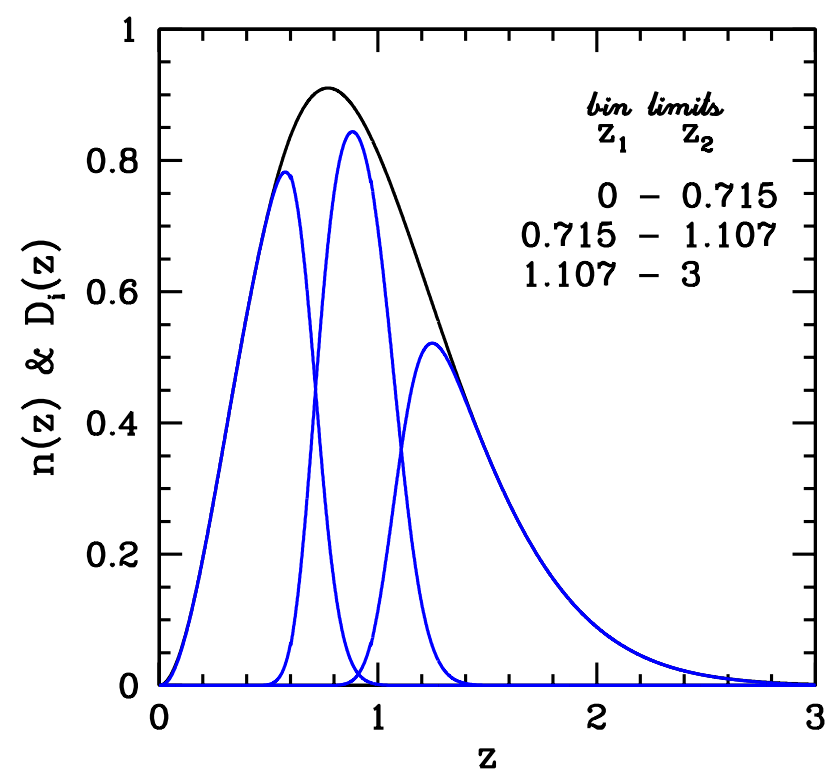

Figure 2. $D_{i}(z)$ distributions in the 3 -bin case. The bin limits $z_{1} \& z_{2}$, shown in the frame, are selected to have the same number of galaxies in each bin. In the 5 -bin case the bin limits $z_{i}$ are 0.560 , $0.789,1.019,1.324,3.00$. The background black line is $n(z)$.

an expression to be used in the next section.

The distribution (2.5) is then considered within the limits of the redshift bins. In order to take into account the discrepancies between the photometric redshift in use, and the actual galaxy redshift, we filter the distributions using window functions $[15,16]$

$$
\begin{aligned}
\Pi_{i}(z) & =\int_{z_{p h, i}}^{z_{p h, i+1}} d z^{\prime} \frac{1}{\sqrt{2 \pi} \sigma(z)} \exp \left(-\frac{\left(z-z^{\prime}\right)^{2}}{2 \sigma^{2}(z)}\right)= \\
& =\frac{1}{2}\left[\operatorname{Erf}\left(z_{p h, i+1}-z\right) / \sqrt{2} \sigma(z)-\operatorname{Erf}\left(z_{p h, i}-z\right) / \sqrt{2} \sigma(z)\right]
\end{aligned}
$$

with $\sigma(z)=\sigma_{z}(1+z), \sigma_{z}=0.05$ (see, e.g., $[17,18]$, for the motivation of this parameter choice).

In figure 2 we show the radial galaxy distribution and the distributions

$$
D_{i}(z)=n(z) \Pi_{i}(z)
$$

in the case of three bins. They must then be normalized, yielding the distributions

$$
\delta_{i}(z)=D_{i}(z) / \int_{0}^{\infty} D_{i}\left(z^{\prime}\right) d z^{\prime}
$$

wherefrom we derive the functions

$$
W_{i}(z)=\frac{3}{2} \Omega_{m} F_{i}(z)(1+z) \quad \text { with } \quad F_{i}(z)=\int_{\Delta z_{i}} d z^{\prime} \delta_{i}\left(z^{\prime}\right) r\left(z, z^{\prime}\right) / r\left(z^{\prime}\right)
$$

shown in figure 3. 


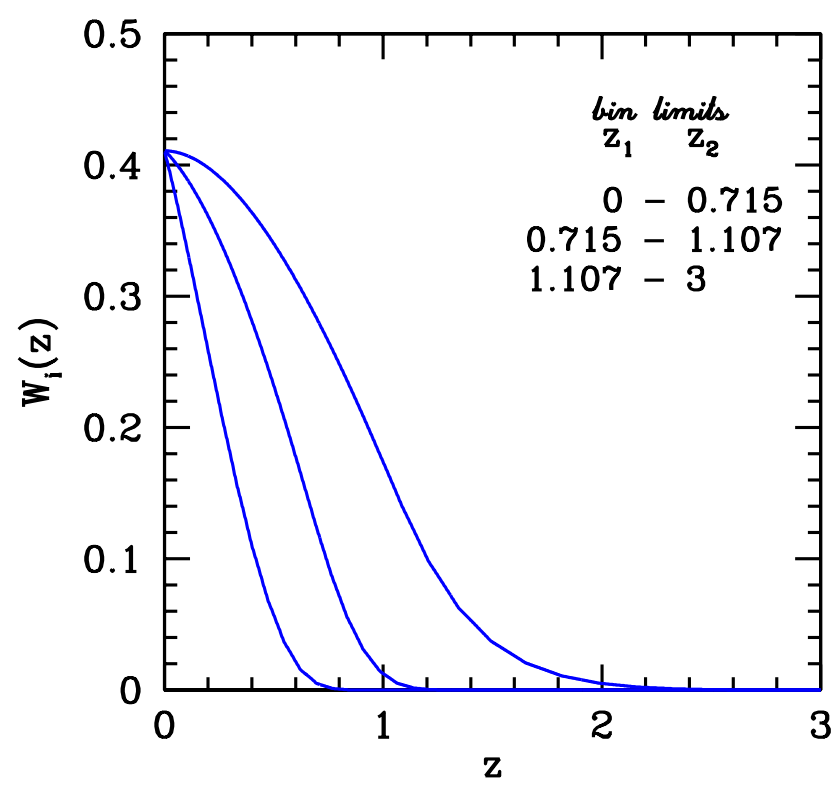

Figure 3. $W_{i}(z)$ window functions adopted for the 3-bin case.

From the power spectrum $P_{i j}(\ell)$, we obtain the covariance matrix

$$
C_{j k}=P_{j k}+\delta_{j k}\left\langle\gamma_{i n t}^{2}\right\rangle n_{j}^{-1}
$$

including the effect of the r.m.s. intrinsic shear $\gamma_{i n t}$, for which we assume $\left\langle\gamma_{\text {int }}^{2}\right\rangle^{1 / 2}=0.22$ $[17] ; n_{j}$ is given by eq. (2.6).

The above expressions enable us to make use of the Fisher matrix formalism, which provides lower limits to the errors on the cosmological parameters one aims to measure. Its basic tool is the likelihood function, yielding the probability that a model, fixed by a set of parameters $p_{\alpha}$, gives the set of data $\mathbf{x}$.

The Fisher matrix for tomographic weak lensing reads [13]

$$
F_{\alpha \beta}=f_{s k y} \sum_{\ell}^{\ell_{\max }} \frac{(2 \ell+1) \Delta \ell}{2} \frac{\partial C_{i j}}{\partial p_{\alpha}} C_{j k}^{-1} \frac{\partial C_{k m}}{\partial p_{\beta}} C_{m i}^{-1} .
$$

The basic ingredients of this expressions are the $C_{i j}$ components; $f_{s k y}$ is the sky fraction covered by the experiment (in our case $f_{s k y}=0.5$ ); the cosmological parameters $p_{\alpha}$ we shall consider are $w_{o}, w_{a}$ and $\Omega_{m}$, so that $\partial / \partial p_{\alpha}$ are partial derivatives with respect to them.

All the other parameters are fixed, in order to keep the number of $N$-body simulations to a manageable level, by assuming data to provide us the other parameters with negligible errors. These parameters include $\Omega_{b}, n_{s}, h$ (baryon density parameter, primeval spectral index of scalar fluctuations, adimensional Hubble parameter), plus a parameter setting the spectral normalization.

If data yield non-linearly evolved amplitudes, it is from them that we should guess suitable initial conditions at $z_{i n}=24$, when starting the simulations, in the linear regime. Here we shall need to improve on the standard normalization procedure which neglects the mild discrepancy between the r.m.s. matter density fluctuation on the comoving scale $R_{8}=$ 
$8 h^{-1} \mathrm{Mpc}$, taken from data, and the linear expression

$$
\sigma_{8, l}^{2}(z)=\left(2 \pi^{2}\right)^{-1} \int_{0}^{\infty} d k A k^{n_{s}+2} \mathcal{T}^{2}(k, z) W^{2}\left(k R_{8}\right)
$$

here $A$ is the primeval fluctuation amplitude at a suitable very high $z_{0}$ value, $\mathcal{T}(k, z)$ is the linear transfer function from such $z_{0}$ value to $z, W(x)$ is a window function, for which we can use the Fourier transform of a top-hat filter: $W(x)=\left(3 / x^{3}\right)(\sin x-x \cos x)$. This mild discrepancy, infact, is $\mathcal{O}(10 \%)$, too much for the signal we are chasing.

Normalizing models to have the same $\sigma_{8, n l}$, as we then need to do, is clearly harder and includes technical aspects which will be further discussed below.

Should we forget this point, and normalize all models so to have the same $\sigma_{8, l}$ at $z=0$, the derivatives with respect to $w_{o}, w_{a}$ or $\Omega_{m}$ are largely dominated by the normalization shift at $z=0$, as the $\sigma_{8, l}-\sigma_{8, n l}$ shift itself depends on $w_{o}, w_{a}$ and $\Omega_{m}$. This would confuse the $z$ dependence of the growth factor, through the observational $z$-range, i.e. the main observable that lensing measures want to exploit.

Once the fiducial models are chosen, the derivatives in eq. (2.12) are evaluated by extracting the power spectra from the simulations of models close to the fiducial ones, obtained by considering parameter increments $\pm 5 \%$.

Our task will then amount to find the regions above a given confidence level. They are $M$-dimensional ellipsoids, about the $M$ parameter values corresponding to fiducial models, that we shall then project onto 2-dimensional subspaces spanned by parameter pairs, so determining constant $\chi^{2}$ contours. In the Fisher Matrix that we adopt, such marginalized likelihood contours will be ellipses centered on the fiducial cosmology.

In a generic case, when the $M$ parameters are dubbed $X_{\alpha}(\alpha=1,2, . ., M)$, the ellipse equation in the $X_{\mu}, X_{\nu}$ plane ( $n=2$ subspace) reads

$$
\left(X_{\mu} X_{\nu}\right)\left[\begin{array}{ll}
F_{\mu \mu}^{-1} & F_{\mu \nu}^{-1} \\
F_{\nu \mu}^{-1} & F_{\nu \nu}^{-1}
\end{array}\right]^{-1}\left(\begin{array}{l}
X_{\mu} \\
X_{\nu}
\end{array}\right)=\Delta \chi^{2}(C L, n) .
$$

Here $\Delta \chi^{2}$ sets the confidence level, being

$$
\Delta \chi^{2}\left(68.3 \%, n_{p}\right)=2.30, \quad \Delta \chi^{2}\left(95.4 \%, n_{p}\right)=4.61,
$$

for $n_{p}=2$.

\section{Cosmological models}

In figure 1 the settings of the fiducial models are shown. The corresponding values of $w_{o}$ and $w_{a}$, are reported in Table 1 . We also take $\Omega_{m}=0.274, h=0.7, \sigma_{8, l}=0.81, n=0.96$ (matter density parameter, Hubble parameter in units of $100 \mathrm{~km} / \mathrm{s} / \mathrm{Mpc}$, linear r.m.s matter fluctuation amplitude at $z=0$ on the scale of $8 h^{-1} \mathrm{Mpc}$, primeval spectral index, respectively) consistently with WMAP7 outputs. We shall discuss below why (slightly) different $\sigma_{8, l}$ values need also be considered.

About the fiducial models we select models characterized by $\pm 5 \%$ increments of $w_{o}$ and $w_{a}$, to perform numerical derivatives. In Table 1 the list of these models is reported.

When $\Lambda \mathrm{CDM}$ is the fiducial model, we consider also derivatives with respect to the $\Omega_{m}$ parameter. In this case all the procedure deals with $\Lambda$ CDM cosmologies; we shall then work out the spectra also for $\Lambda$ CDM models with $\Omega_{m}=0.2603$ and 0.2877 . 


\begin{tabular}{cccccc}
\hline \multicolumn{2}{c}{$\Lambda \mathrm{CDM}$} & \multicolumn{2}{c}{ M1 } & \multicolumn{2}{c}{$\mathrm{M} 3$} \\
\hline \hline$w_{0}$ & $w_{a}$ & $w_{0}$ & $w_{a}$ & $w_{0}$ & $w_{a}$ \\
-1 & 0 & -0.67 & -2.28 & -1.18 & 0.89 \\
-1.05 & 0 & -0.7035 & -2.28 & -1.239 & 0.89 \\
-0.95 & 0 & -0.6365 & -2.28 & -1.121 & 0.89 \\
-1 & +0.05 & -0.67 & -2.394 & -1.18 & 0.9345 \\
-1 & -0.05 & -0.67 & -2.166 & -1.18 & 0.8455 \\
\hline
\end{tabular}

Table 1. List of values of $w_{o}$ and $w_{a}$ used to perform numerical derivatives.

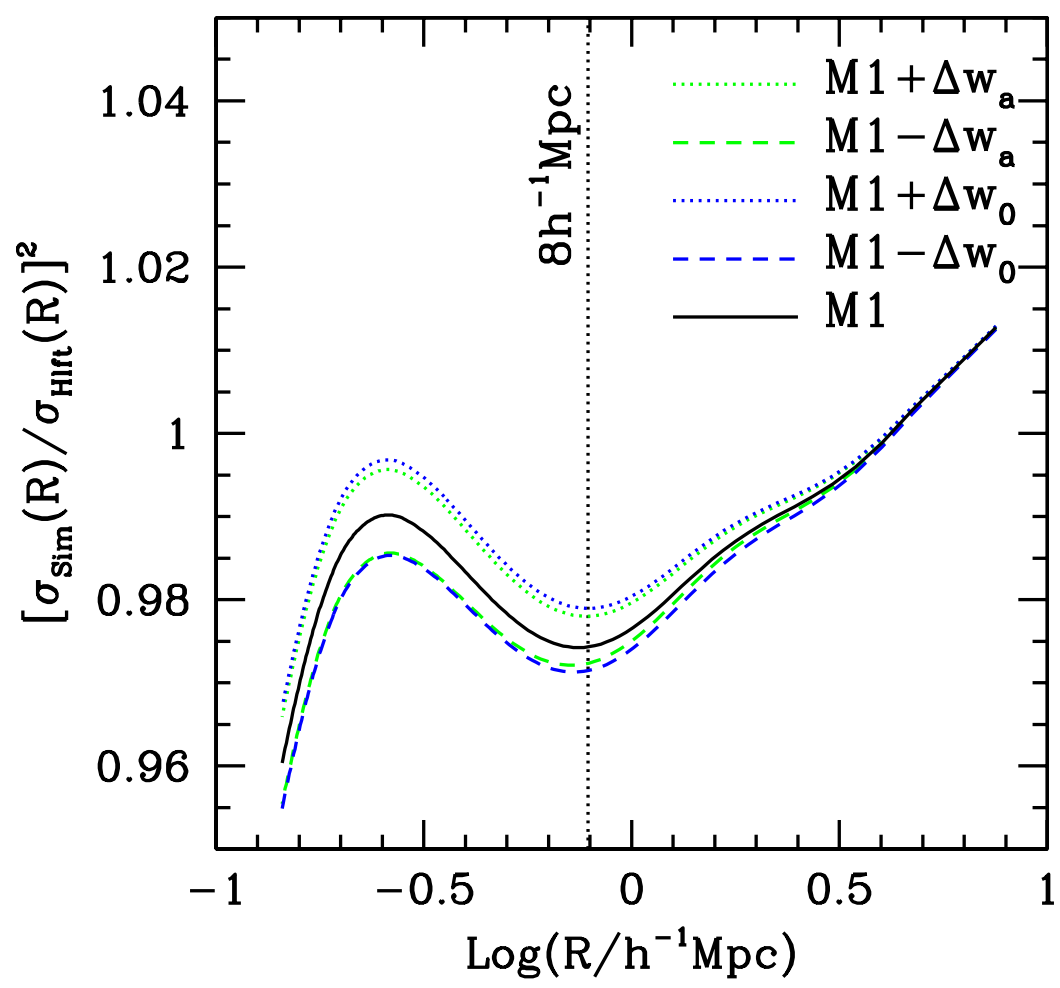

Figure 4. $R$-dependence of the ratio between the $\sigma^{2}(R)$ 's at $z=0$ obtained from simulations, for models in the neighborhood of M1; they are normalized to the respective mass variances obtained using HALOFIT, however almost identical for all these models (see text).

For $\Lambda \mathrm{CDM}$ and all nearby models, two different initial seeds were also considered, to test the dependence on initial conditions, finding that Fisher Matrix results are almost insensitive to it. Accordingly, for the other fiducial models, only one seed is used. Altogether we run 24 model simulations.

\section{Simulations and their analysis}

$N$-body simulations are performed by using a modified version of PKDGRAV [19] able to handle any DE state equation $w(a)$. To this aim, $N^{3}=256^{3}$ particles, representing CDM and baryons, are set in a box with side $L=256 h^{-1} \mathrm{Mpc}$. Particle masses are therefore $m_{c}=$ 


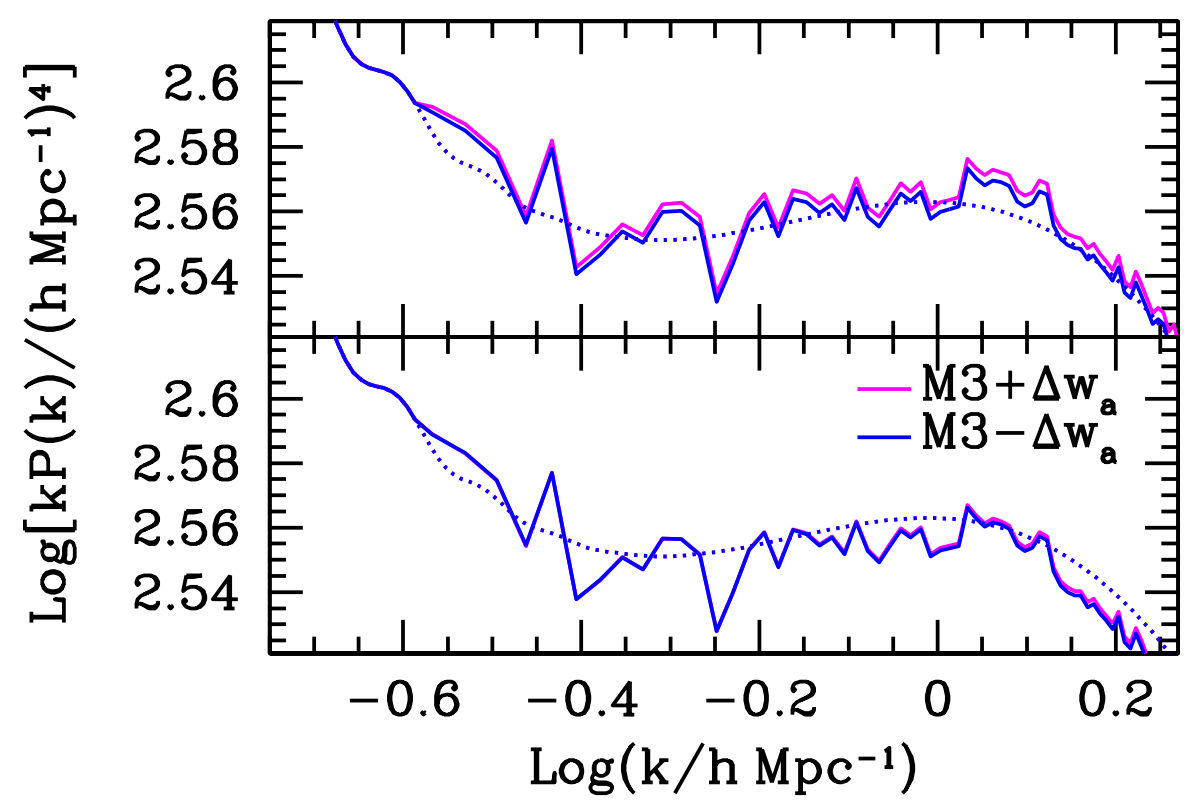

Figure 5. Connection between HALOFIT and simulation spectra at the low $-k$ end. The upper (lower) panel shows the resulting spectra when normalizing to $\sigma_{8, l}(z=0)\left(\sigma_{8, n l}(z=0)\right)$. The dotted line is HALOFIT.

$\rho_{o, c r} \Omega_{m}(L / N)^{3}=7.61 \times 10^{10} h^{-1} M_{\odot}\left(\rho_{o, c r}\right.$ : critical density). Softening is $1 / 40$ of the initial intra-particle distance, yielding $\epsilon \simeq 25 h^{-1} \mathrm{kpc}$ (wavenumber $\kappa=2 \pi / \epsilon \simeq 150 h \mathrm{Mpc}^{-1}$ ).

Transfer functions generated using the CAMB package are employed to create initial conditions, with a modified version of the PM software by Klypin and Holzmann [20], also able to handle suitable parameterizations of DE. Simulations are started at $z_{i n}=24$. The initial density field is obtained from the same random numbers for all the simulations. For the $\Lambda$ CDM fiducial we generate each simulation with two sets of initial random numbers.

Matter power spectra are obtained by performing a FFT (Fast Fourier Transform) of the matter density fields, that we computed from the particles distribution, through a Cloud-inCell algorithm, by using a regular grid with $N_{g}=2048$. This allows us to obtain non-linear spectra in a large $k$-interval.

In particular, our resolution allows to work out spectra up to $k \simeq 10 \mathrm{hpc}^{-1}$. However, for $k>2-3 h \mathrm{Mpc}^{-1}$ neglecting baryon physics is no longer accurate [21]. Although both simulations and HALOFIT refer to purely gravitational dynamics, we wish to examine only physically significant scales. Therefore, we consider WL spectra for $\ell<2000$ only, so limiting the contribution of scales $k>2 h \mathrm{Mpc}^{-1}$ below $\simeq 5 \%$, at most.

Let us now discuss the normalization problem. In Figure 4 we show the dependence on scale of the r.m.s. of matter density fluctuations (mass variance), as obtainable through the expression

$$
\sigma^{2}(R)=\sum_{k} \Delta k_{k} P(k) k^{2} W^{2}(k R)
$$

where $P(k)$ is the full non-linear spectrum. When applied to simulations, we denote the variance as $\sigma_{\text {sim }}^{2}(R)$. Plotting the scale dependence for $\sigma_{\text {sim }}^{2}(R)$, rather than for the simulation spectra $P_{\text {sim }}(k)$, smears out the numerical oscillations that spectra unavoidably exhibit. 
Figure 4 allows us to focus on the discrepances between the model M1 and the nearby models used to perform derivatives about it. The spectra shown in the figure were obtained by normalizing initial conditions so that their $\sigma_{8, l}$ at $z=0$ coincide. As a consequence the whole linear spectra of M1 and nearby models (not shown in the Figure) are nearly coincident, at $z=0$, since the models share all parameters except the DE state equation, which has a very weak impact on the linear spectrum shape. If one uses HALOFIT to correct the linear spectra, the $z=0$ non-linear spectra would still coincide, since HALOFIT only depends on $\sigma_{8, l}$ and on $\Omega_{d e}$, not on $w$. In contrast, Figure 4 confirms that the simulation spectra differ. Accordingly, taking the Fisher matrix derivatives fixing $\sigma_{8, l}$ is different from fixing $\sigma_{8, n l}$. Besides of the points made above, here we choose to normalize to the same non-linear variance $\sigma_{8, n l}$ also because this is the primary observational quantity and we expect that future data will put direct constraints on it, resulting in a strong prior in the Fisher matrix.

The price to pay is that producing simulations with different cosmological parameters but coincident $\sigma_{8, n l}$ is not a trivial task and requires a trial-and-error search of initial conditions for the $N$-body simulations.

There is then another question, concerning the low $-k$ spectrum. The point is that the integration (2.1) unavoidably extends down to $k$ 's which are still close to (or fully in) the linear regime. In principle, dealing with such scales should not create difficulties. However, the spectra obtained from our simulations, run in a box of $256 h^{-1} \mathrm{Mpc}$, become increasingly noisy and discrete when the linear regime is approached. To solve this problem we extrapolate our numerical spectra with the linear theoretical ones at scales larger than a certain threshold.

The problem is then where and how to connect numerical spectra with theoretical ones. Let then be $k_{p}=2 p \pi /\left(256 h^{-1} \mathrm{Mpc}\right)$, being $p$ an integer; we use simulation spectra when $\Delta k_{p} / k_{p}$ shifts below 0.1 , i.e. for $\log \left(k / h \mathrm{Mpc}^{-1}\right)>-0.61$. At lower $k$ 's HALOFIT spectra are used.

Figure 5 (upper frame) is the result of this operation, when done using $M 3 \pm \Delta w_{a}$ spectra characterized by the same $\sigma_{8, l}(z=0)$. Spectral shifts of this kind affect the ratios between derivatives with respect to cosmological parameters (in this case, $w_{a}$ ). In the same Figure (lower frame) we show the result of the connection after renormalizing spectra to the same $\sigma_{8, n l}$. Some scale dependent discrepancy still remains, but the main signal comes then from the different redshift dependence of model spectra, rather than from the shift among $\sigma_{8, n l}$.

\section{Predictions on parameter errors}

\subsection{Derivatives}

The next step is evaluating the derivatives in eq. (2.12). For the sake of illustration we plot the results of such differentiation in the case of a single bin, by considering: (i) the spectra obtained from simulations, as illustrated in previous section; (ii) HALOFIT spectra; (iii) purely linear spectra.

In figure 6 we show the derivatives and, in the lower frame, compare the results of the cases (i) and (ii), when the fiducial model is $\Lambda$ CDM. The results obtained starting simulations from two different seeds are also compared. In figure 7 we similarly show derivatives when the fiducial model is M1 or M3. One seed only is considered there, and we consider the parameters $w_{0}$ and $w_{a}$ only.

The effects of BAO's as well of irregularities in simulation spectra are clearly visible in all plots. There is however a clear difference between the derivative with respect to $\Omega_{m}$, 

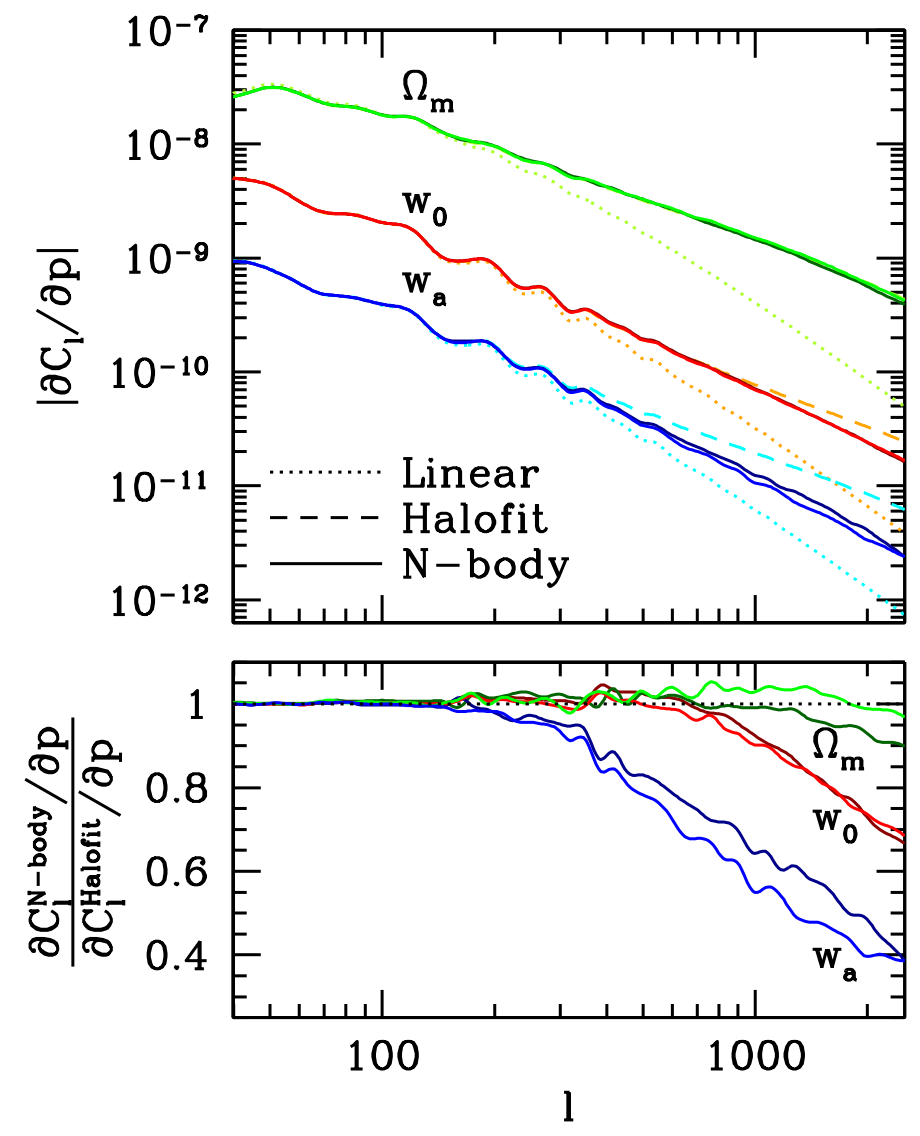

Figure 6. Derivatives of $C_{\ell}$, in the case of a single bin, for $20<\ell<2000$, with respect to the parameters $\Omega_{m}, w_{0}$ and $w_{a}$, when the fiducial model is $\Lambda$ CDM. In the upper frame we show their different behavior, for $N$-body simulations, HALOFIT and linear spectra. In the lower frame we show the ratio between derivatives obtained from $N$-body simulations and HALOFIT. For these models we run simulations with two different seeds. Derivatives obtained from each of them are in the same colors, using a darker tonality for the seeds considered just for these models.

in figure 6 , and any other one. For both seeds the ratio between its value for simulations and HALOFIT is close to unity, with a discrepancy exceeding $\sim 5 \%$ just for $\ell>1500$, when contributions from $k$ values above the range of HALOFIT validity become non-irrelevant. This is a further confirmation of the coherence between $N$-body simulations and HALOFIT, for $\Lambda$ CDM models, the only ones involved in the differentiation.

Still in figure 6, we notice that differences between seeds mostly stays within $6-7 \%$, even in the case of the parameter $w_{a}$, which is the most sensitive to noise effects.

The case M1, shown in figure 7, exhibits then a peculiarity, with respect to all other cases. Here, the ratios of derivatives with respect to $w_{0}$ and $w_{a}$ almost overlap up to $\ell \simeq 500$, while their trend remains similar even up to $\ell=1000$. The effect is peculiar of M1, and recurs also when more bins are considered. For $\Lambda \mathrm{CDM}$, the behaviors of the two ratios are significantly discrepant. In the case M3, finally, the two ratios exhibit neatly different behaviors. This reflects onto the shape of the likelihood ellipses which are one of the main results of our work. 

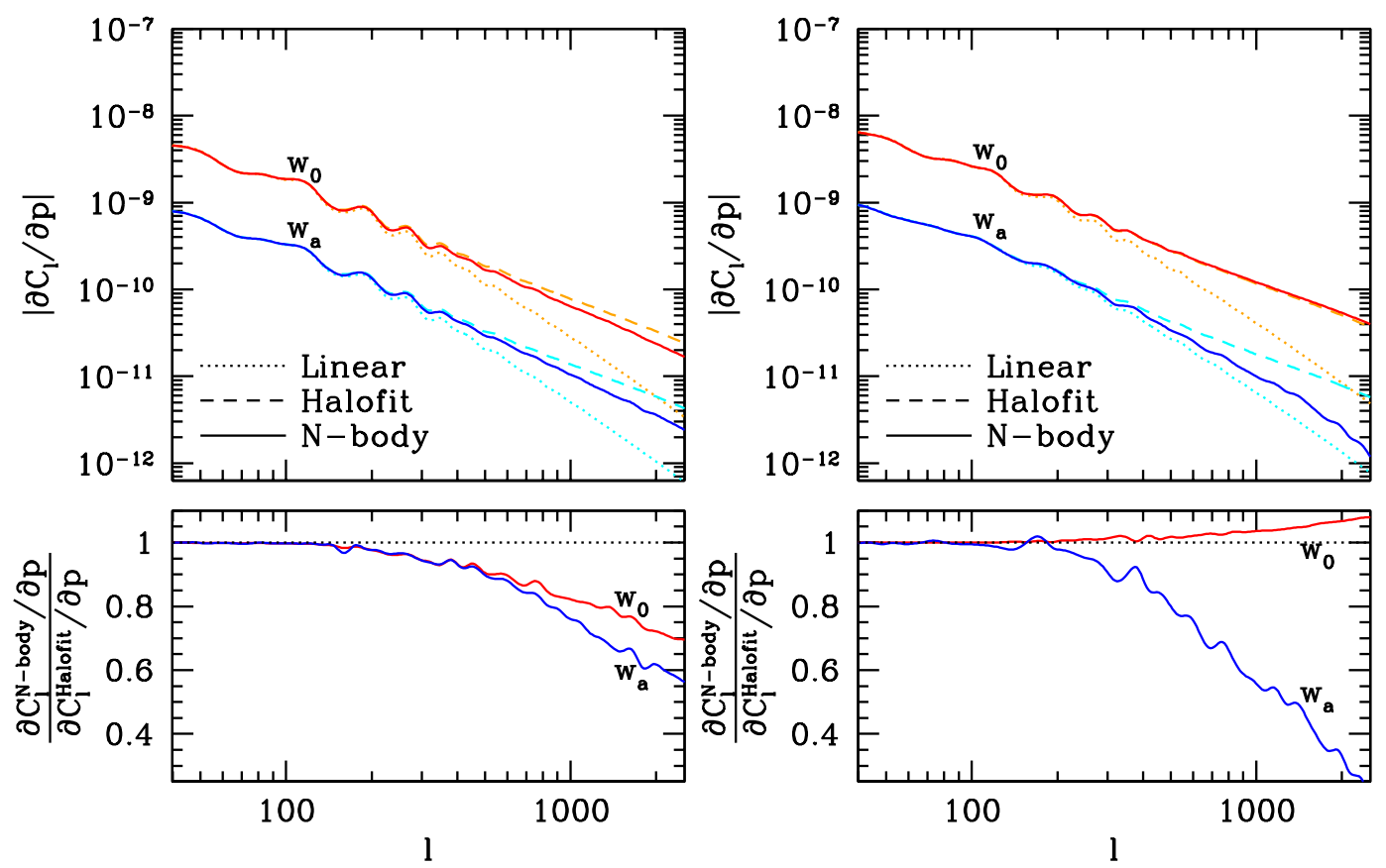

Figure 7. Derivatives of $C_{\ell}$, as in the previous figure, for M1 and M3 fiducial models (1.h.s. \& r.h.s., respectively), omitting the parameter $\Omega_{m}$, which is not considered in these cases.

\subsection{Likelihood ellipses: $\Lambda$ CDM neighborhood}

In figures 8 and 9 we show the confidence ellipses, when the fiducial model is $\Lambda \mathrm{CDM}$, in the cases of 3 or 5 bins and using $C_{\ell}$ up to $\ell=500,1000$ or 2000 .

Up to $\ell \simeq 500$, i.e. including just a mildly non-linear scale range, discrepancies between HALOFIT and simulations already indicate an underestimate of errors in the HALOFIT case. Discrepancies approach a factor 2 when we go up to $\ell=1000$, both for 3 and 5 bins. They become quite significant in the $\ell=2000$ case. In the latter cases we observe a modification of the likelihood contours, indicating a different correlation between the errors on different parameters. This includes the $w_{a}-\Omega_{m}$ correlation and means that a neglect of the degrees of freedom of DE state equation leads to errors on the estimate of the density parameter $\Omega_{m}$.

Meanwhile, the discrepancy between simulations started from different seeds are much smaller. Different seeds yield discrepancies still visible in derivatives (see figure 6), but the integrations needed to pass from them to likelihood ellipses smear them out. In fact, the shaping of the ellipses is dominated by the $z$-dependence of the spectral behavior, and here is where HALOFIT is apparently unable to meet simulation results. The changes in the confidence ellipses are also quantified in Table 2.

Incidentally, let us notice that the improvements, when passing from the 3 - to the 5 -bin case, are more significant when a greater $\ell$ is considered.

In particular, in Table 2, we give the inverse area of the ellipses at 95\% CL ; when considered in the $w_{0}-w_{a}$ plane, it coincides with the figure of merit

$$
F O M=\frac{1}{\operatorname{area}(95 \% \mathrm{CL})}=\frac{\sqrt{\operatorname{Det}\left|F_{i j}\right|}}{4.61 \pi}
$$




\begin{tabular}{cccc}
\hline & & HALOFIT & sim. average \\
\hline \hline$w_{0}-w_{a}$ & $\theta$ & $-71.4^{\circ}$ & $-70.1^{\circ}$ \\
plane & axial ratio (95\% CL) & 3.27 & 6.22 \\
& $1 /$ area(95\% CL) & 50.2 & 43.4 \\
& correlation & -0.69 & -0.88 \\
\hline$w_{0}-\Omega_{m}$ & $\theta$ & $1.90^{\circ}$ & $1.71^{\circ}$ \\
plane & axial ratio (95\% CL) & 39.3 & 75.7 \\
& $1 /$ area $(95 \% \mathrm{CL})$ & $3.22 \times 10^{3}$ & $3.92 \times 10^{3}$ \\
& correlation & 0.79 & 0.92 \\
\hline$\Omega_{m}-w_{a}$ & $\theta$ & $-0.15^{o}$ & $-0.49^{\circ}$ \\
plane & axial ratio (95\% CL) & 54.7 & 104 \\
& $1 /$ area $(95 \% \mathrm{CL})$ & $8.73 \times 10^{2}$ & $8.19 \times 10^{2}$ \\
& correlation & -0.14 & -0.67 \\
\hline \hline standard & $\sigma_{w_{o}}$ & $2.90 \times 10^{-2}$ & $3.65 \times 10^{-2}$ \\
deviations & $\sigma_{w_{a}}$ & $6.57 \times 10^{-2}$ & $9.36 \times 10^{-2}$ \\
& $\sigma_{\Omega_{m}}$ & $1.21 \times 10^{-3}$ & $1.18 \times 10^{-3}$ \\
\hline
\end{tabular}

Table 2. Features of likelihood ellipses in the neighborhood of $\Lambda \mathrm{CDM}$, for the 5 bin case with $\ell=2000$; the angle between the major axis and the abscissa is dubbed $\theta$; the abscissa being $w_{0}$ (former two cases) or $w_{a}$. For the definition of the inverse area and correlation see text.

introduced in the report of the Dark Energy Task Force [22]; the factor 4.61 yields 95\% CL (see eq. (2.15)).

The correlation coefficient or Pearson correlation measures the correlation between two variables $X, Y$. In terms of Fisher Matrix components, it reads

$$
\rho(X, Y)=F_{X Y}^{-1} / \sqrt{F_{X X}^{-1} \times F_{Y Y}^{-1}} .
$$

Table 2 confirms that, when considering models different from $\Lambda \mathrm{CDM}$, non linear correction obtained through HALOFIT may be misleading. This is true even when the fiducial model is $\Lambda$ CDM itself and we just consider mild deviations of $w$ from -1 . Let us point out, in particular, the $w_{a}-\Omega_{m}$ case; here the parameter correlation increases by a factor $\sim 5$, when passing from HALOFIT to simulations, because of a radical change in the orientation of the ellipse, as the angle between its major axis and the $w_{a}$ axis passes from $-0.15^{\circ}$ to $-0.49^{\circ}$ (see also figure 9).

As expected, the error on $\Omega_{m}$ estimate is not affected by the passage from simulations to HALOFIT; in this case we are dealing with $\Lambda$ CDM models only. On the contrary, using HALOFIT leads to underestimates of the errors on $w_{0}$ and $w_{a}$, by a substantial $30-40 \%$. Let us however outline that the substantial shift of the $\Omega_{m}-w_{a}$ correlation means that the very estimate of the matter density parameter $\Omega_{m}$ is in jeopardy, as soon as we admit mild deviations from pure $\Lambda \mathrm{CDM}$, so that the density of $\mathrm{DE}$ has a slight dependence on redshift.

\subsection{Confidence ellipses: M1 \& M3 models}

Figures 10 and 11 then show the results in the $w_{0}-w_{a}$ plane, when the fiducial models are M1 or M3. It is evident that the two cases are quite different. This reflects the behaviors of derivatives, whose ratios almost overlap in the M1 case, while remaining different in the M3 case. 

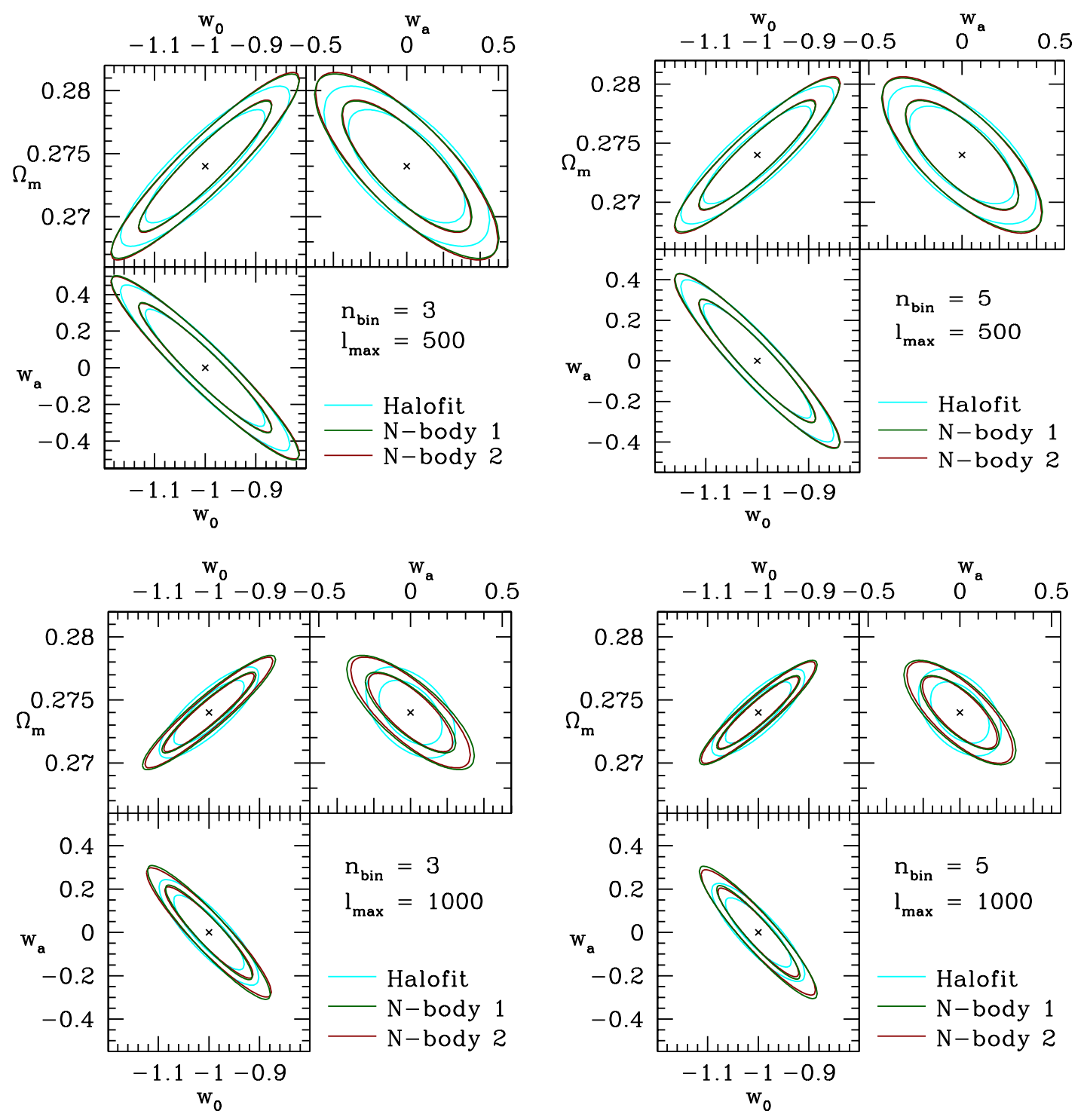

Figure 8. Likelihood ellipses, showing $65 \%$ and $95 \%$ CL contours, for all parameter pairs, including signals up to either $\ell \simeq 500$ or 1000 for the $\Lambda \mathrm{CDM}$ fiducial. In the former case no significant discrepancy between HALOFIT and simulation results can be appreciated. Discrepancies become more relevant in the latter case, both for 3 and 5 bins. Notice also that the two simulation seeds yield almost overlapping outputs.

In the M1 case, we see just quite a mild shift. For $\ell=500$ or 1000, and 3 or 5 bins, the discrepancy appears rather small, being just slightly more evident for $\ell=2000$. Let us however outline that such mild discrepancies are $\mathcal{O}(10 \%)$, compatible with discrepancies attaining $\sim 20 \%$ in the derivatives (see figure 7 , left panel). It is also clear that including larger $\ell$ 's means integrating over slightly greater discrepancies. The key issue, however, is that, until the two derivative ratios proceed with similar trends, the only effect on ellipses is a change in their area, while the orientation of their axes does not change.

Let us now come to the M3 case, where the derivative ratios exhibit radically differ- 

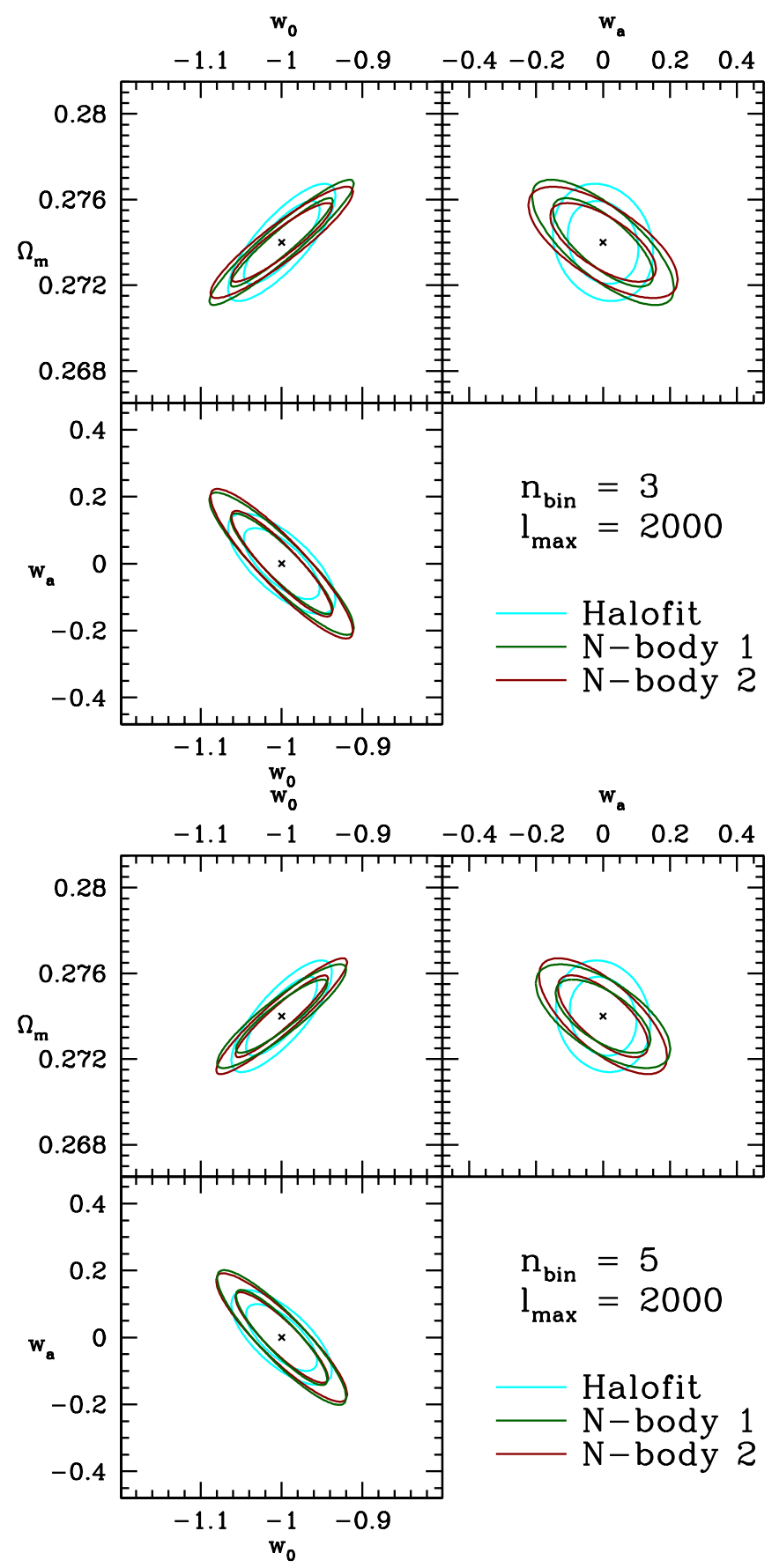

Figure 9. Likelihood ellipses, showing $65 \%$ and $95 \%$ CL contours, for all parameter pairs, considered including signals up to $\ell \simeq 2000$ for the $\Lambda$ CDM fiducial. Here simulations and HALOFIT yield significantly different outputs.

ent trends. The most immediate effect is that errors estimated through HALOFIT exceed simulation errors by a substantial factor. Discrepancies are already visible when only mildly non-linear contributions are included and increase sharply when exploiting more deeply nonlinear spectral areas. 

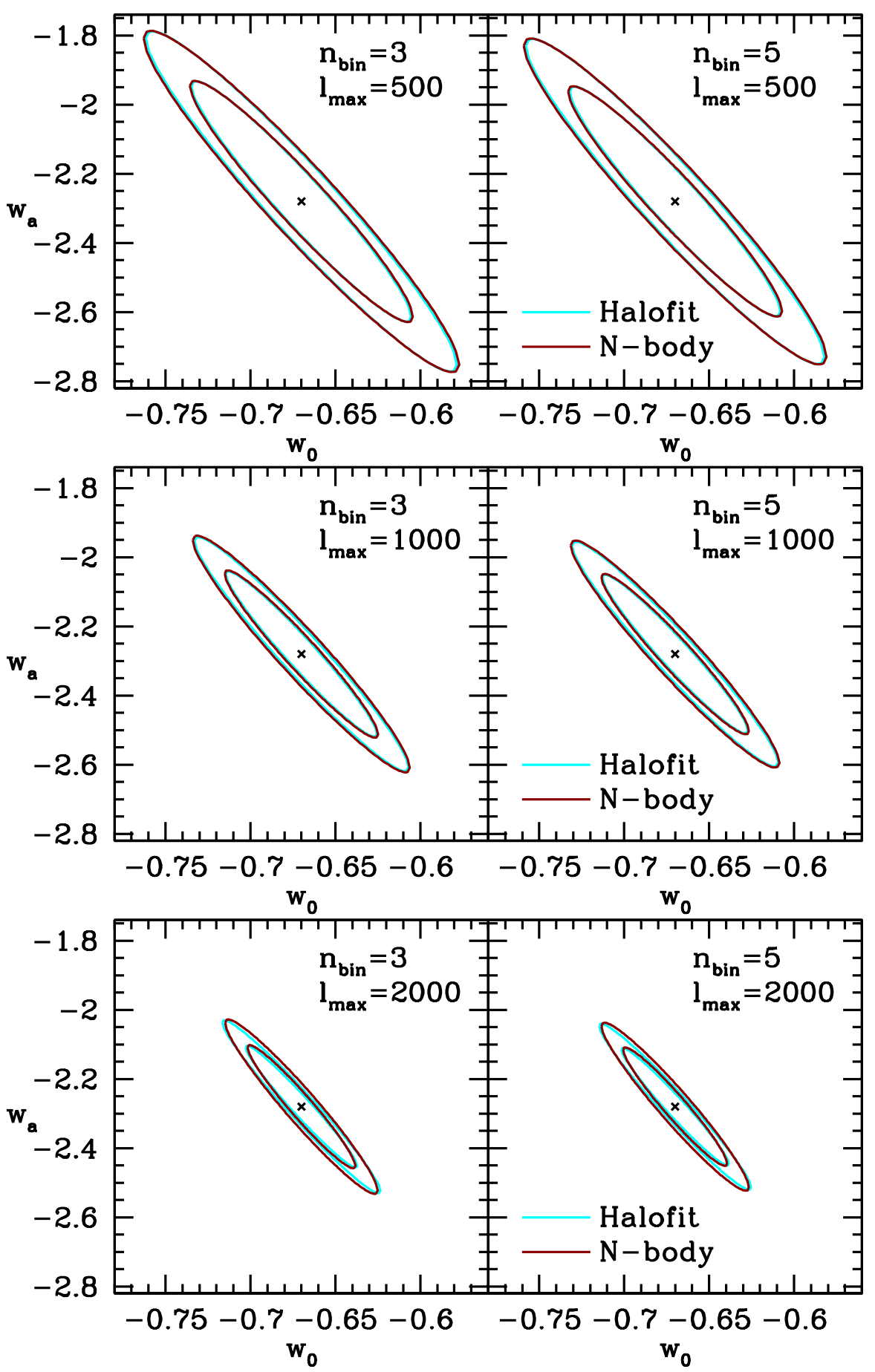

Figure 10. Contours at $68 \%$ and $95 \%$ CL for the M1 model. HALOFIT ellipses almost overlap with the $N$-body ones.

In Table 3 we report quantitative information on the deformation of likelihood ellipses on the 5 -bin $\ell=2000$ case. The only elements which do not change much are the correlation and, accordingly, the angle $\theta$. On the contrary, the ratio between the axes of the ellipses and 


\begin{tabular}{cccc}
\hline & & HALOFIT & sim. average \\
\hline \hline$w_{o}-w_{a}$ & $\theta$ & $-80.6^{\circ}$ & $-83.9^{\circ}$ \\
plane & axial ratio $(95 \% \mathrm{CL})$ & 49.7 & 18.3 \\
& $1 /$ area $(95 \% \mathrm{CL})$ & 181 & 505 \\
& correlation & -0.99 & -0.89 \\
\hline \hline standard & $\sigma_{w_{o}}$ & $2.26 \times 10^{-2}$ & $0.6 \times 10^{-2}$ \\
deviations & $\sigma_{w_{a}}$ & 0.136 & $4.98 \times 10^{-2}$ \\
\hline
\end{tabular}

Table 3. Features of likelihood ellipses when the fiducial model is M3, for the 5 bin case with $\ell=2000$; symbols as in Table 2 .

the FOM vary by a factor $\sim 2.5$. Standard deviations are also deeply affected.

Altogether, this is a case when estimates based on HALOFIT are simply not trustworthy.

\section{Conclusions}

In the last decades, cosmological observations led to the most important discoveries in fundamental physics. The dark cosmic components are either a proof of physics beyond the standard model of elementary interactions or a way to parametrize violations of General Relativity. In the former case, the two dark components could be the phenomenological description of a single cosmic fluid [23]. Energy exchanges between such components could then be a signal of such dark unity [24]. In turn, this opens a list of possibilities which is embarrassingly long.

This florilegium of options is a clear consequence of the lack of data. Tomographic weak lensing measures will clearly contribute to provide more of them. This undoubtedly implies an important experimental effort and there are scarce doubts that, accordingly, data interpretation tools deserve to be updated.

In this work we outline the need to improve our capacity to predict the spectra of cosmological models. When we restrict ourselves within the frame of $\Lambda$ CDM cosmologies, e.g. to perform derivatives in respect to $\Omega_{m}$, the HALOFIT expressions still yield acceptable outputs (see Figure 6). Improving their accuracy keeps however a need, namely for the parameter range consistent with most recent observations.

A first technical conclusion is that an inspection of the non-linear part of spectra, through tomographic weak lensing surveys, greatly improves their discriminatory power. This is also confirmed by the effects of including an increasing number of $\ell$ harmonics in the analysis: when HALOFIT and simulations yield discrepant results, the difference between them increases when including greater and greater $\ell$ values. On the contrary, with the instrumental features assumed here, essentially corresponding to those of the EUCLID [11] project, there seems to be no clear advantage in going from 3 to 5 bins.

Our main conclusion is however that, going beyond a DE state equation with $w \equiv-1$, direct model simulation remains the only efficient way to obtain spectra. Some attempt to generalize HALOFIT to constant $w \neq-1$, although important, do not hit the heart of the problem, as we rather expect a variable $w(z)$. We should also be aware of the severe bias on $w(z)$ detection which could follow the assumption of constant $w$ [25].

In this work we have tested the effects of using $\Lambda$ CDM-suited HALOFIT expressions, when the true cosmology is a simple dDE model, straightforwardly consistent with WMAP7 data [2]. 

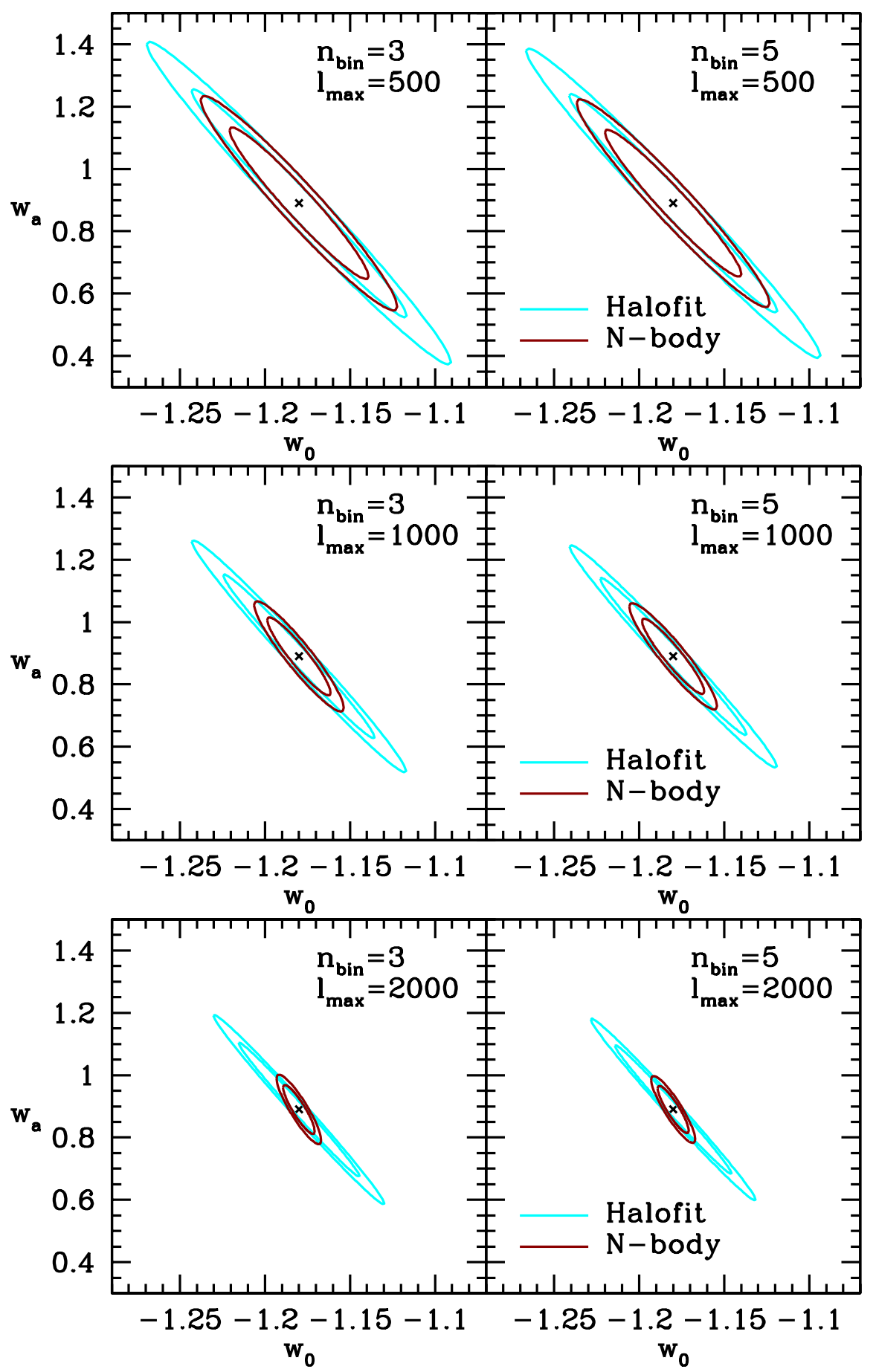

Figure 11. Contours at $68 \%$ and $95 \%$ CL for the M3 model.

We find that, even with $\Lambda \mathrm{CDM}$ as fiducial, when trying to estimate the error on $w_{0}$ and $w_{a}$, we could be misled, if using HALOFIT expressions. Not only they lead there to error misestimates, but they can also imply a wrong correlation between the estimate of $\Omega_{m}$ and, e.g., $w_{a}$. Henceforth, the very $\Omega_{m}$ estimate is in jeopardy, if we do not treat accurately 
the functional ensemble of state equations $w(z)$ about $w \equiv-1$. This is, perhaps, the most striking result of this work.

We extended our test to the two dynamical DE models, differing from $\Lambda$ CDM just for $w(z)$, which are however within $95 \%$ CL from $\Lambda$ CDM, on the basis of available data. Curiously enough, the two models behave rather differently.

For one of them, the discrepancies between HALOFIT and simulations, although visible in the derivatives, leave just $\mathcal{O}(10 \%)$ discrepancies in similarly oriented likelihood ellipses. In this case error estimates performed through HALOFIT turn out to be fair.

In the case of the other fiducial model, we meet an absolutely different situation. First of all, using HALOFIT leads to error overestimates by large factors (100 to $200 \%$ larger), to a wrong figure of merit, and to other problems which can be summarized by stating that HALOFIT-based results are wrong.

In the latter cases (non- $\Lambda \mathrm{CDM}$ ) we have not yet tested the intercorrelation between DE state equations and the density parameter. This will be among our future tasks.

In spite of that, these results suggest a trend in the effectiveness of HALOFIT, when non$\Lambda \mathrm{CDM}$ cosmologies are involved. We meet: (i) An apparent reliability of such expressions, when the fiducial model is characterized by negative $w_{a}$. (ii) Significant difficulties for the $\Lambda \mathrm{CDM}$ case $\left(w_{a}=0\right)$, where we expected them to have their best performance. (iii) In the case of positive $w_{a}$, finally, we conclude that HALOFIT expressions yield misleading results.

We might then conjecture that an indicator of HALOFIT efficiency is the sign of the derivative

$$
w^{\prime}(0)=\frac{d w}{d z}(z=0) .
$$

When $w^{\prime}(0) \geq 0$, any use of HALOFIT expressions seems dangerous. However, as we have been dealing only with DE state equations which can be fully characterized at $z=0$, this conjecture need to be tested for other cases.

Altogether, let us conclude that HALOFIT, in its present form, is not adequate to deal with the degrees of freedom opening when non $-\Lambda \mathrm{CDM}$ cosmologies are considered, as none can predict, a priori, which can be the physical $w(z)$ behavior.

\section{Acknowledgments}

Most numerical simulations were performed on the PIA and PanStarrs2 clusters of the MaxPlanck-Institut für Astronomie at the Rechenzentrum in Garching. Spectral analysis was performed at CINECA-Bologna under the CINECA-INAF agreement (2008-2010). The financial supports of MIUR through the PRIN08 program and of the Italian Space Agency (ASI), though the COFIS program are acknowledged. L. A. acknowledges support from the DFG project TRR33 "The Dark Universe".

\section{References}

[1] M. M. Phillips, Astrophys. J. 413 L105 (1993); A. G. Riess; R. P. Kirshner, B. P. Schmidt, S. Jha, et al., Astron. J. 116, 1009 (1998); S. Perlmutter, G. Aldering, G. Goldhaber, et al.; Astrophys. J. 517, 565 (1999); then see also, e.g. A. G. Riess; M. Kowalski et al. Astrophys. J. 686, 749 (2008); M. Hicken, W. M. Wood-Vasey, S. Blondin, P. Challis, S. Jha, P. L. Kelly, A. Rest, R. P. Kirshner, Astrophys. J. 700, 1097 (2009); R. Kessler et al., Astrophys. J. S. 185, 32 (2009)

[2] E. Komatsu et al. Astrophys. J. Suppl. , 180, 330 (2009) 
[3] B. A. Reid et al. , Mon. Not. R. Aston. Soc. 404, 60 (2010); W. J. Percival, S. Cole, D. J. Eisenstein, R. C. Nichol, J. A. Peacock, A. C. Pope and A. S. Szalay, Mon. Not. R. Aston. Soc. 381, 1053 (2007)

[4] E. V. Linder and R. N. Kahn, Astropart.Phys. 28, 481 (2007)

[5] C. di Porto and L. Amendola, Phys. Rev. D 77, h3508 (2008)

[6] A. Refregier, A. Amara, T. D. Kitching, A. Rassat, R. Scaramella, J. Weller et al. [arXiv:1001.0061]

[7] D. Huterer, Phys. Rev. D 65, 063001 (2002); D. Huterer and M. Takada, Astropart. Phys. 23 369 (2005)

[8] R. E. Smith, J. A. Peacock, A. Jenkins, S. D. M. White, C. S. Frenk, F. R. Pearce, P. A. Thomas, G. Efstathiou and H. M. P. Couchman, Mon. Not. R. Aston. Soc. 341, 1311S (2003)

[9] S. Hilbert, J. Hartlap, S. D. M. White, P. Schneider, Astronom. Astrophys. 499, 31 (2009); K. Heitmann et al. Astrophys. J. 715, 104 (2010)

[10] P. McDonald, H. Trac and C. Contaldi, Mon. Not. R. Aston. Soc. 366547 (2006)

[11] A. Cimatti, R. Laureijs, B. Leibundgut, S. Lilly, R. Nichol, A. Reefregier, P. Rosati, M. Steinmetz, N. Thatte, E. Valentijn ESA-SRE (2009) [arXiv:0912.0914]

[12] T. Schrabback et al. Astronom. Astrophys. , 516, 63 (2010)

[13] W. Hu and B. Jain, Phys. Rev. D 70, 043009 (2004)

[14] W. Hu, Astrophys. J. 522 L21 (1999)

[15] W. Hu, Z. Ma and D. Huterer, Astrophys. J. 636, 21 (2006)

[16] G. Bernstein and Z. Ma, Astrophys. J. 682, 39 (2008)

[17] A. Amara and A. Refregier, Mon. Not. R. Aston. Soc. 3811018 (2007)

[18] L. Amendola, M. Kunz, D. Sapone, JCAP 0804, 013 (2008)

[19] J. G. Stadel, "Cosmological N-Body Simulations and Their Analysis", Ph.D. thesis, University of Washington, Pullman, 2001

[20] A. Klypin, J. Holtzman (1997) [arXiv:astro-ph/9712217]

[21] Y. P. Jing, P. Zhang, W. P. Lin, L. Gao, V. Springel, Astrophys. J. 640, L119 (2006); D. H. Rudd, A. R. Zentner, A. V. Kravtsov, Astrophys. J. 672, 19 (2008); L. Casarini, A. V. Macciò, S. A. Bonometto, G. Stinson, Mon. Not. R. Aston. Soc. (2010, in press) [arXiv:1005.4683]; A. R. Zentner et al, Phys. Rev. D 77, 043507 (2008); A. P. Hearin and A. R. Zentner, JCAP 04, 32 (2009)

[22] A. Albrecht et al., "Report of the Dark Energy Task Force" (2006) [arXiv:astro-ph/0609591]

[23] R. Mainini, L.P.L. Colombo, S. A. Bonometto, Astrophys.J. 632, 691 (2005); M. C. Bento, O. Bertolami, A. A. Sen, Gen.Rel.\&Gr. 35, 2063 (2003)

[24] L. Amendola, Phys. Rev. D 62, 3511 (2000); D. Comelli, M. Pietroni and A. Riotto, Phys. Lett. B 571, 115 (2002)

[25] L. Casarini, A. V. Macciò, S. A. Bonometto, JCAP, 3, 14 (2009); L. Casarini, JCAP 8,5 (2009) 